Conference abstract PDD30

\title{
Membrane-Mediated Effects of General Anesthetics on Ion Channels
}

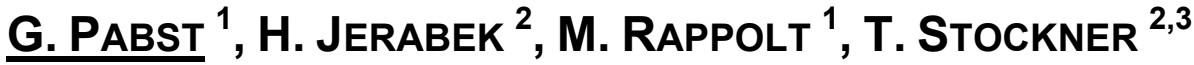 \\ ${ }^{1}$ Institute of Biophysics and Nanosystems Research, Austrian Academy of Sciences, Graz, Austria \\ 2 Department of Health \& Environment, Austrian Institute of Technology, Seibersdorf, Austria \\ ${ }^{3}$ Department of Medical Chemistry, Medical University of Vienna, Vienna, Austria \\ E-mails: georg.pabst@oeaw.ac.at (G. Pabst), thomas.stockner@meduniwien.ac.at (T. Stockner) \\ Sci Pharm. 2010; 78: 619 \\ doi:10.3797/scipharm.cespt.8.PDD30
}

General anesthetic drugs were introduced more than 160 years ago and are indispensable in daily surgery at hospitals. Interestingly, their mode of action remains largely unresolved. At present, there are two schools, one favoring specific (direct) interactions of the drugs with proteins of the central nervous system and a second adhering to nonspecific mechanisms through a modulation of biophysical membrane properties. We have focused on the latter mechanism and studied the effects of R-(-), S-(+)-ketamine and propofol on the biophysical properties of lipid model membranes composed of palmitoyl oleoyl phosphatidylcholine by a combination of X-ray diffraction and all-atom molecular dynamics simulations. In agreement with several previous studies, we do not find significant changes to the overall membrane structure up to $8 \mathrm{~mol} \%$ drug content. However, we observed that the insertion of drugs within the lipid/water interface caused significant changes of lateral pressures within the membrane [1]. The changes are predicted to be stereospecific and large enough to affect the opening probability of ion channels at concentrations that compare remarkably well to clinically applied concentrations. Our results thus provide a novel basis for rational anesthetic drug design.

[1] Jerabek H, Pabst G, Rappolt M, Stockner T. Membrane-mediated effect on ion channels induced by the anesthetic drug ketamine. J Am Chem Soc. 2010; 132:7990-7997. doi:10.1021/ja910843d 